\section{Clinical benefit of sunitinib in gastrointestinal stromal tumors with different exon 11 mutation genotypes}

\author{
Zhi Dong', Jing Gao', Jifang Gong', Jie Li', Yanyan Li', Lin Shen' \& Jian Li,1
}

\begin{abstract}
Aim: To assess the efficacy of second-line sunitinib therapy in gastrointestinal stromal tumor patients with different exon 11 mutation genotypes. Patients \& methods: Thirty eight of the 75 patients received imatinib (IM) dose escalation followed by sunitinib (IM escalation group), while 37 were switched to sunitinib directly after the failure of first-line IM treatment (sunitinib group). Progression-free survival and overall survival were compared. Results: The median progression-free survival in the sunitinib group was significantly longer than in the IM escalation group (14 vs 4 months; $p<0.001$ ), so was in patients with exon 11 deletions (16 vs 3 months; $p<0.001$ ). Conclusion: Patients who have an exon 11 deletion mutation are more likely to benefit from switching to sunitinib directly than from IM dose escalation.
\end{abstract}

First draft submitted: 1 June 2017; Accepted for publication: 26 June 2017;

Published online: 7 July 2017

Gastrointestinal stromal tumor (GIST) is the most common sarcoma of the GI tract, accounting for $0.1-3 \%$ of malignant GI tumors [1]. In patients diagnosed with GIST, functional gene mutations of c-kit and PDGFRA ( $\alpha$ polypeptide) are responsible for pathogenesis of the malignancy in $80-85 \%$ and $5-7 \%$ of cases, respectively [2]. Currently, the standard first-line therapy recommended for the treatment of advanced or metastatic GIST is imatinib (IM), a small molecule tyrosine kinase inhibitor of $c-k i t$ and PDGFRA genes [3]. However, the majority of patients with advanced GIST develop disease progression within 6 months (primary resistance) or after 2 years (secondary resistance) of initial IM treatment $[4,5]$.

In Phase III clinical trials, $30 \%$ of patients with GIST who fail on standard-dose IM therapy have been found to benefit from IM dose escalation [6]. Other studies have shown that administration of the multitargeted receptor tyrosine kinase inhibitor sunitinib malate significantly improves progression-free survival (PFS) and overall survival (OS) after disease progression on IM has occurred, especially in $c$-kit exon 9 mutant or wild-type GIST $[7,8]$. Exon 11 is the most common site of $c$-kit mutations in GIST ( $70 \%$ of tumors) and the deletion mutation has been shown to be associated with a poor prognosis compared with other mutation types such as point mutations [9]. However, the relationship between exon 11 mutation types and the efficacy of sunitinib is still unknown. Although a retrospective study has shown a significant efficacy difference between deletion and nondeletion mutation types in patients who received IM dose escalation [10], this has not yet been demonstrated with sunitinib therapy.

The aim of this retrospective study was to analyze the clinical efficacy of second-line sunitinib therapy in Chinese patients with advanced or metastatic GIST who have different exon 11

'Laboratory of Carcinogenesis \& Translational Research for the Ministry of National Education, Department of Gl Oncology,

Peking University School of Oncology, Beijing Cancer Hospital \& Institute, Beijing, 100142 China

*Author for correspondence: Tel.: +86 10 88196561; Fax: +86 10 88196561; oncogene@163.com

\section{KEYWORDS}

- exon 11 deletion mutation - gastrointestinal stromal tumors $\bullet$ imatinib dose escalation $\bullet$ sunitinib

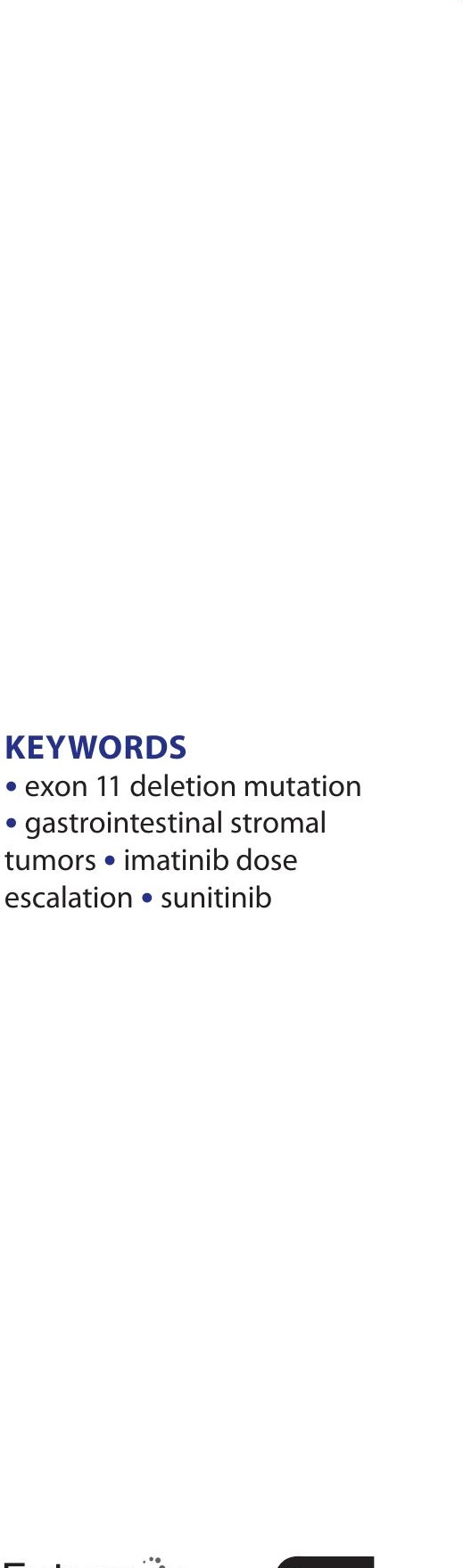


mutation genotypes, and also to compare the survival benefit of IM dose escalation followed by sunitinib treatment with switching to sunitinib directly in patients with different exon 11 mutation genotypes.

\section{Materials \& methods}

\section{- Patients \& study design}

The records of 963 patients with advanced or metastatic GIST who received treatment at Beijing Cancer Hospital between 2003 and 2013 were analyzed retrospectively to identify patients suitable for inclusion in the study. The last follow-up date in the records analyzed was December 2015. Approval for the study was given by the Beijing Cancer Hospital Ethics Committee.

The main inclusion criteria for the selection of patients for the study were: a confirmed diagnosis of GIST with disease progression after initial IM treatment; receipt of sunitinib treatment following the occurrence of progression on first-line IM therapy; appropriate clinical data for analysis and data on $c$-kit and PDGFRA gene mutations in primary tumor tissues; evaluation of clinical efficacy at least once after the initiation of sunitinib treatment; and availability of complete follow-up data. The main exclusion criteria were: receipt of sunitinib treatment for less than 1 month; irregular clinical follow-ups; and absence of $c$-kit and PDGFRA gene mutation results.

\section{- Exon 11 mutation status}

We classified exon 11 mutation status into two types: deletion mutation and nondeletion mutation. Deletion mutation included deletion mutation and insertion-deletion mutation; nondeletion mutation included other mutation types except deletion and insertion-deletion mutation types.

\section{- Treatments}

The patients selected for analysis were in two groups: the IM escalation group in whom the IM dosage was escalated to $600 \mathrm{mg} /$ day after failure of the initial dosage of $400 \mathrm{mg} /$ day and subsequent sunitinib treatment (at a dosage of either $50 \mathrm{mg} /$ day on a 4 weeks on, 2 weeks off basis or $37.5 \mathrm{mg} /$ day continuously) following the occurrence of further disease progression; and the sunitinib group in whom treatment was switched to sunitinib directly (at the same dosages as in the IM escalation group) after failure of the initial IM dosage of $400 \mathrm{mg} /$ day.

\section{- Efficacy evaluation}

Patients were evaluated for clinical efficacy every 6 weeks via the Response Evaluation Criteria in Solid Tumors. Responses were classified as having either a complete response, partial response (PR), stable disease (SD) or progressive disease (PD).

\section{- Study end points}

The primary study end point was the PFS of GIST patients with different exon 11 mutation genotypes who received second-line sunitinib treatment. Secondary end points included the efficacy of IM escalation therapy in GIST patients with different exon 11 mutation types; and differences in PFS and OS of GIST patients with different exon 11 mutation types who received either IM dose escalation followed by sunitinib treatment on further disease progression or sunitinib treatment directly following the failure of first-line IM therapy.

\section{- Statistical analysis}

All data were analyzed by the SPSS ${ }^{\circledR} 21.0$ platform (SPSS, Inc., IL, USA). Medians and frequencies were used for group variables and a chi-square test was performed to examine the differences in PFS and OS between the treatment groups. PFS and OS curves were generated via Kaplan-Meier methodology and a log-rank test was used to compare the differences. A p $<0.05$ was considered statistically significant.

\section{Results}

\section{- Patient characteristics}

Data on a total of 75 patients with advanced or metastatic GIST were selected for analysis. All patients analyzed were treated with IM as the first-line therapy (400 mg/day) and had experienced tumor progression. The median PFS with first-line IM therapy was 32 months. The IM escalation group, who received IM dose escalation followed by sunitinib on further disease progression comprised 38 patients, while the sunitinib group who were switched directly to sunitinib after the failure of first-line IM therapy comprised 37 patients.

The total patient group included 60 male and 15 female patients, and the median age was 55 years (range: $29-74$ years). The primary lesions were mostly detected in the intestines $(34.3 \%)$, stomach $(32.0 \%)$ and abdominopelvic cavity $(12.0 \%)$. More than $80 \%$ of the patients underwent primary tumor resection 


\begin{tabular}{|c|c|c|c|c|}
\hline Characteristic & IM escalation, $\mathrm{n}(\%)^{\dagger}$ & Sunitinib, $\mathrm{n}(\%)^{\dagger}$ & Total, n (\%) ${ }^{\dagger}$ & p-value \\
\hline Number of patients & 38 & 37 & 75 & \\
\hline Age, years (median/range) & $55 / 29-74$ & $52 / 38-71$ & $53 / 29-74$ & 0.734 \\
\hline Sex (male/female) & $31 / 7$ & $29 / 8$ & $60 / 15$ & 0.692 \\
\hline \multicolumn{5}{|l|}{ Primary site: } \\
\hline - Stomach & 15 (39.5\%) & $9(24.3 \%)$ & $24(32 \%)$ & \\
\hline - Duodenum & $4(10.5 \%)$ & $4(10.8 \%)$ & $8(10.7 \%)$ & \\
\hline - Small intestine & $13(34.2 \%)$ & $12(32.5 \%)$ & $25(34.3 \%)$ & \\
\hline - Omentum/mesentery & $2(5.3 \%)$ & $2(5.4 \%)$ & $4(5.3 \%)$ & \\
\hline - Abdominopelvic cavity & $4(10.5 \%)$ & $5(13.5 \%)$ & $9(12.0 \%)$ & \\
\hline - Colorectum & 0 & $5(13.5 \%)$ & $5(6.7 \%)$ & \\
\hline Radical surgery: & & & & 0.503 \\
\hline - Yes & $33(86.8 \%)$ & $30(81.1 \%)$ & $63(84 \%)$ & \\
\hline$-\mathrm{No}$ & $5(13.2 \%)$ & $7(18.9 \%)$ & $12(16 \%)$ & \\
\hline \multicolumn{5}{|l|}{ IM adjuvant therapy: } \\
\hline - Yes & $5(13.2 \%)$ & $3(8.1 \%)$ & $8(10.7 \%)$ & \\
\hline- No & $5(13.2 \%)$ & $5(13.5 \%)$ & $10(13.3 \%)$ & \\
\hline - Unknown & $28(73.6 \%)$ & $29(78.4 \%)$ & $57(76 \%)$ & \\
\hline Outcome with first-line IM therapy: & & & 0.352 & \\
\hline - Complete response & $6(15.8 \%)$ & $2(5.4 \%)$ & $8(10.7 \%)$ & \\
\hline - Partial response & $17(44.7 \%)$ & $18(48.6 \%)$ & $35(46.7 \%)$ & \\
\hline - Stable disease & $12(31.6 \%)$ & $14(37.8 \%)$ & $26(34.7 \%)$ & \\
\hline - Progressive disease & $3(7.9 \%)$ & $3(8.2 \%)$ & $6(8 \%)$ & \\
\hline \multicolumn{5}{|l|}{ Recurrence sites ${ }^{\ddagger}:$} \\
\hline - Liver & $30(51.7 \%)$ & $25(43.1 \%)$ & $55(47.4 \%)$ & \\
\hline - Peritoneum & $2(3.4 \%)$ & $2(3.4 \%)$ & $4(3.4 \%)$ & \\
\hline - Local recurrence & $2(3.4 \%)$ & $1(1.7 \%)$ & $3(2.6 \%)$ & \\
\hline - Abdominopelvic cavity & $20(34.6 \%)$ & $27(46.7 \%)$ & $47(40.5 \%)$ & \\
\hline - Stomach & $3(5.2 \%)$ & 0 & $3(2.6 \%)$ & \\
\hline - Adrenal & 0 & $1(1.7 \%)$ & $1(0.9 \%)$ & \\
\hline - Colorectum & 0 & $2(3.4 \%)$ & $2(1.7 \%)$ & \\
\hline - Bone & $1(1.7 \%)$ & 0 & $1(0.9 \%)$ & \\
\hline Primary mutation type: & & & & 0.334 \\
\hline - Exon 11 deletion & $22(57.9 \%)$ & $22(59.5 \%)$ & $44(58.7 \%)$ & \\
\hline - Exon 11 nondeletion & $9(23.7 \%)$ & $12(32.4 \%)$ & $21(28 \%)$ & \\
\hline - Exon 9 & $5(13.2 \%)$ & $1(2.7 \%)$ & $6(8 \%)$ & \\
\hline - Exon 17 & $1(2.6 \%)$ & 0 & $1(1.3 \%)$ & \\
\hline - Wild-type & $1(2.6 \%)$ & $2(5.4 \%)$ & $3(4 \%)$ & \\
\hline \multicolumn{5}{|l|}{ Secondary mutation type: } \\
\hline - Exon 17 & $7(18.4 \%)$ & $3(8.1 \%)$ & $10(13.3 \%)$ & \\
\hline - Exon 11 & 0 & $1(2.7 \%)$ & $1(1.3 \%)$ & \\
\hline - Exon 13 & $5(13.1 \%)$ & $3(8.1 \%)$ & $8(10.7 \%)$ & \\
\hline - Wild-type & $2(5.3 \%)$ & $3(8.1 \%)$ & $5(6.7 \%)$ & \\
\hline - Unknown & $24(63.2 \%)$ & $27(73 \%)$ & $51(68 \%)$ & \\
\hline \multicolumn{5}{|c|}{$\begin{array}{l}\text { IM escalation group, imatinib dose escalation followed by sunitinib after further disease progression; sunitinib group, switch to } \\
\text { sunitinib directly after failure of standard-dose imatinib therapy. } \\
\text { 'Except where otherwise stated. } \\
\text { \$2 } 20 \text { patients in the IM group and } 21 \text { in the sunitinib group had more than two metastatic lesions. } \\
\text { IM: Imatinib. }\end{array}$} \\
\hline
\end{tabular}

before receiving IM therapy. The most common metastatic sites were the liver $(47.4 \%)$ and abdominopelvic cavity (40.5\%).
The response rate with first-line IM treatment was $60.5 \%$ in the IM escalation group and $54 \%$ in the sunitinib group. In 65 patients, exon 11 
mutations were detected in the primary lesions; $44(58.7 \%)$ of these patients had a deletion mutation and $21(28.0 \%)$ had a nondeletion mutation. Other genotypes included an exon 9 mutation in six patients, an exon 17 mutation in one, and a wild-type in three. Following disease
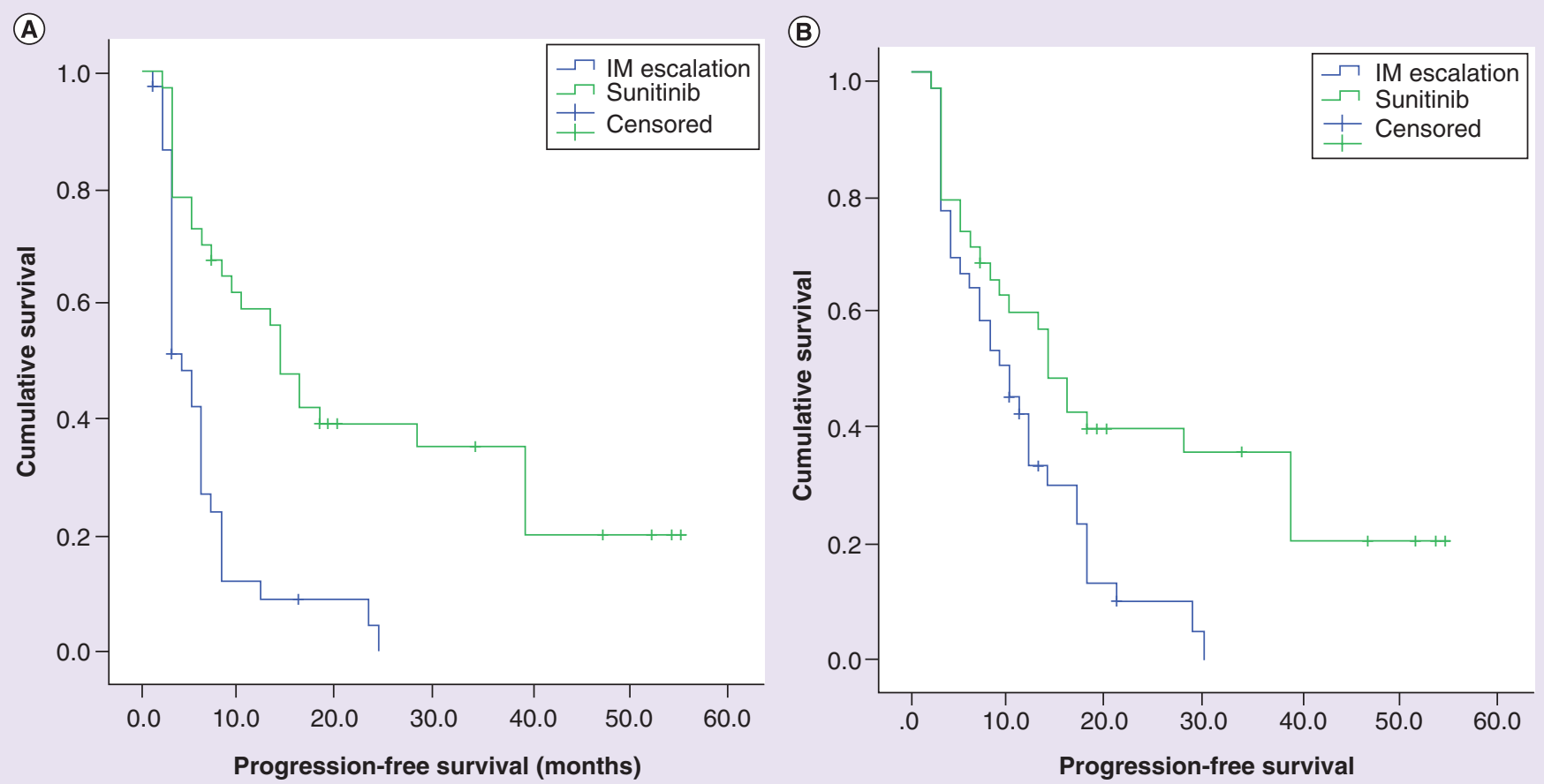

(c)

(D)
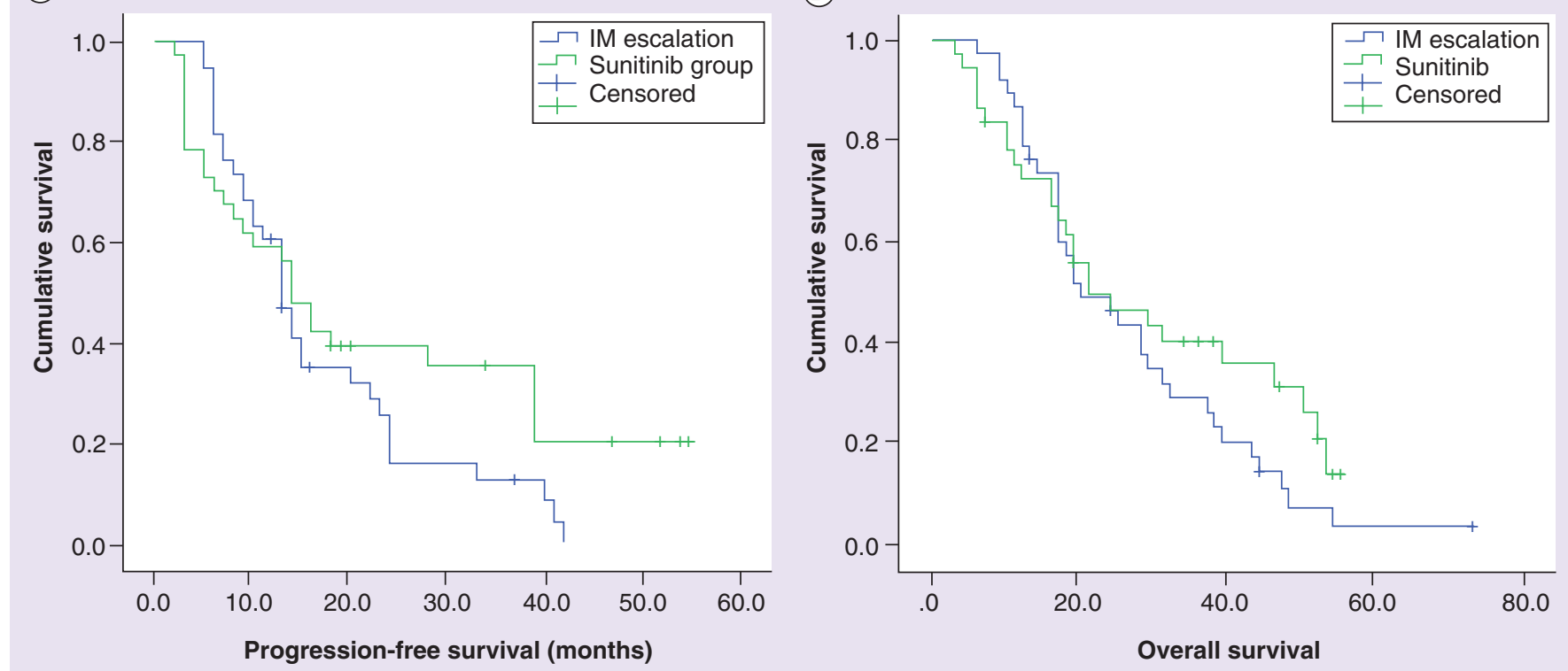

Figure 1. Kaplan-Meier curves in the imatinib escalation and sunitinib groups. (A) Kaplan-Meier curves for PFS in the IM escalation to $600 \mathrm{mg} /$ day period and in the sunitinib group. (B) Kaplan-Meier curves for PFS with a shift to sunitinib therapy after IM escalation to $600 \mathrm{mg} /$ day period and in the sunitinib group. (C) Kaplan-Meier curves for PFS in the IM escalation group including escalation to $600 \mathrm{mg} /$ day and subsequent sunitinib treatment and in the sunitinib group. (D) Kaplan-Meier curves for overall survival in the IM escalation and sunitinib groups.

IM: Imatinib; PFS: Progression-free survival. 
progression with first-line IM treatment, secondary mutations were detected in 24 patients, including an exon 17 mutation in 10 and an exon 13 mutation in 8 (Table 1).

\section{- Clinical outcomes}

Clinical benefit of IM dose escalation followed by sunitinib versus a switch to sunitinib directly after failure of first-line IM therapy In the IM escalation group, 17 patients achieved SD (44.7\%) and 21 had PD (55.3\%). In the sunitinib group, 1 patient had a PR $(2.7 \%)$, 28 had SD (75.7\%) and 8 had PD (21.6\%) after switching to sunitinib therapy. The disease control rate (i.e., complete response $+\mathrm{PR}$ $+\mathrm{SD})$ in the two groups was 44.7 and $78.4 \%$, respectively ( $\mathrm{p}=0.002$ ) (Table 2 ). Meanwhile, there was significant difference in disease control rate of both groups with the presence of secondary resistance mutation (35.7 vs $80 \%$; $\mathrm{p}<0.001)$. To be specific, five patients had SD and nine had PD in IM escalation group, while eight had SD and two had PD in sunitinib group. Over a median follow-up period of 56 months, the median PFS in the sunitinib group was significantly longer than in the IM escalation to $600 \mathrm{mg} /$ day period (14 months; $95 \%$ CI: $10.5-17.5$ vs 4 months; 95\% CI: $2.9-5.1$; p $<0.001)$ and a shift to sunitinib therapy after IM escalation period (14 months; $95 \% \mathrm{CI}$ : 10.5-17.5 vs 9 months; 95\% CI: 5.4-12.6; p $=0.006$ ) in IM escalation group (Figure 1A \& B) However,there was no significant difference in PFS between switching to sunitinib directly (14 months; 95\% CI: 10.5-17.5) and the total PFS with initial IM dose escalation and subsequent sunitinib therapy (13 months; 95\% CI: 10.515.5; $\mathrm{p}=0.187$; Figure $1 \mathrm{C}$ ). And there was no significant difference in the OS between the two patient groups (21 months; 95\% CI: 7.7-34.3 vs 20 months; 95\% CI: 11.8-28.2, respectively; $\mathrm{p}$ $=0.230$ ) (Figure 1D).
Clinical efficacy in patients with different mutation genotypes

Among the 65 patients with exon 11 mutations, those in the sunitinib group $(n=34)$ had a longer median PFS than patients in the IM escalation group $(\mathrm{n}=31)$ (14 vs 3 months, respectively; $\mathrm{p}<0.001)$. In the six patients with an exon 9 mutation, the median PFS in the two patient groups was 12 months (95\% CI: 3.4-20.6) and 17 months (95\% CI: 0-38.5), respectively. The single patient in the sunitinib group with an exon 9 mutation had no disease progression at the end of follow-up and a PFS of 7 months.

One patient with $c$-kit/PDGFRA wild-type had a PFS of 3 months with IM escalation therapy and 12 months with subsequent sunitinib treatment, and the OS in this patient was 19 months. In two patients in the sunitinib group with a wildtype phenotype, the PFS was 5 and 14 months, respectively, and the OS was 19 and 21 months, respectively. The single patient with an exon 17 mutation had a PFS of 3 months with both IM escalation therapy and subsequent sunitinib treatment.

Clinical efficacy in patients with different exon 11 mutation genotypes

In the IM escalation group, 22 patients had an exon 11 deletion mutation and 9 had an exon 11 nondeletion mutation. No significant difference in PFS was found between patients with the two mutation types (3 months; 95\% CI: $2.5-3.5$ vs 5 months; 95\% CI: 3.6-6.4, respectively; $\mathrm{p}=$ $0.927)$. In the sunitinib group, 22 patients had an exon 11 deletion mutation and 12 had an exon 11 nondeletion mutation. As in the IM escalation group, the differences in PFS between patients with the two mutation types was not statistically significant (16 months; $95 \% \mathrm{CI}$ : 1.5-30.5 vs 9 months; $95 \% \mathrm{CI}: 5.6-12.4 ; \mathrm{p}=$ $0.291)$.

Table 2. Disease control rate in the imatinib escalation and sunitinib groups.

\begin{tabular}{|c|c|c|c|c|c|c|}
\hline \multirow[t]{2}{*}{ Group } & \multicolumn{4}{|c|}{ Patients, n (\%) } & \multirow[t]{2}{*}{ DCR (\%) } & \multirow[t]{2}{*}{$p$-value } \\
\hline & CR & PR & SD & PD & & \\
\hline $\begin{array}{l}\text { IM escalation } \\
\text { group }\end{array}$ & 0 & 0 & 17 (44.7\%) & $21(55.3 \%)$ & $44.7 \%$ & 0.002 \\
\hline $\begin{array}{l}\text { Sunitinib } \\
\text { group }\end{array}$ & 0 & $1(2.7 \%)$ & $28(75.7 \%)$ & $8(21.6 \%)$ & $78.4 \%$ & \\
\hline
\end{tabular}


(A)

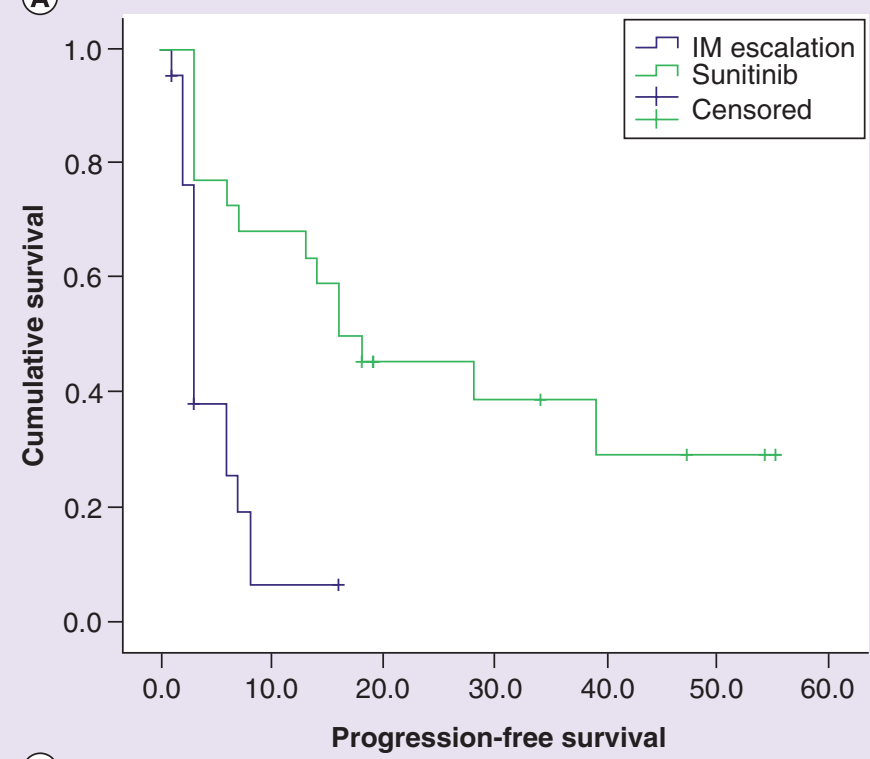

(C)

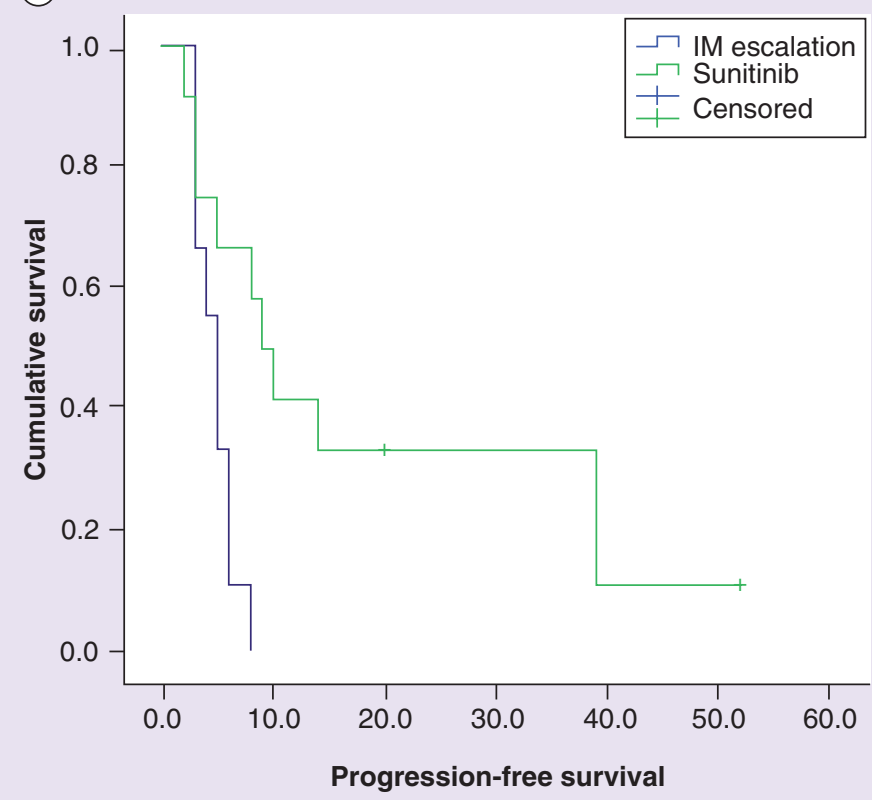

(B)

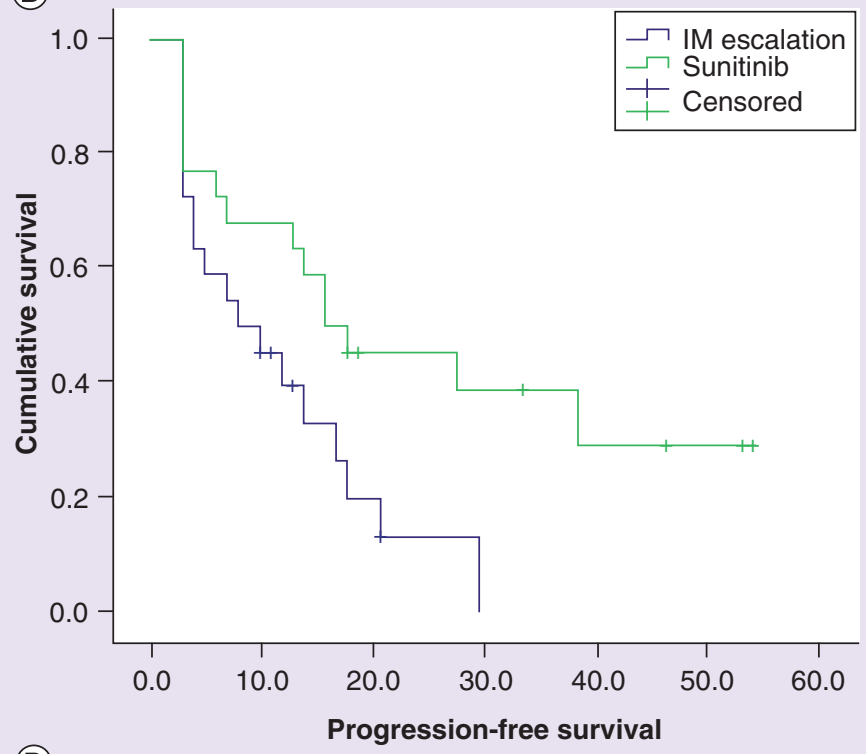

(D)

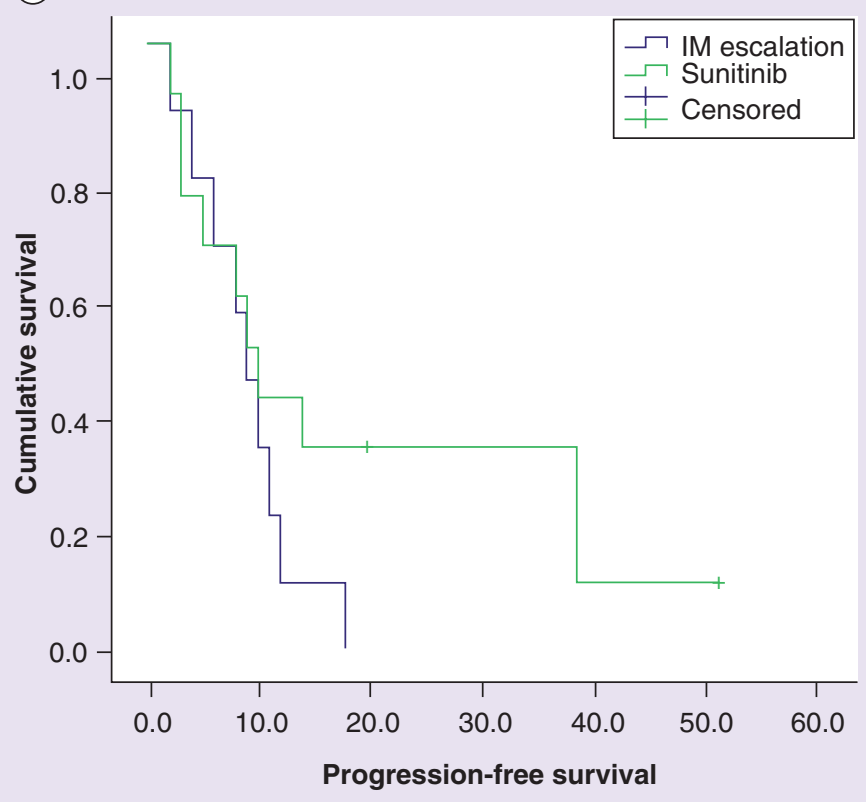

Figure 2. Kaplan-Meier curves in the IM escalation and sunitinib groups with different exon 11 mutation types. (A) Kaplan-Meier curves for PFS in the IM escalation to $600 \mathrm{mg} /$ day period and sunitinib group in patients with an exon 11 deletion mutation. (B) KaplanMeier curves for PFS with subsequent sunitinib after IM escalation to $600 \mathrm{mg} /$ day and a switch to sunitinib directly in patients with an exon 11 deletion mutation. (C) Kaplan-Meier curves for PFS in the IM escalation to $600 \mathrm{mg} /$ day and sunitinib group in patients with an exon 11 nondeletion mutation. (D) Kaplan-Meier curves for PFS with subsequent sunitinib after IM escalation to $600 \mathrm{mg} / \mathrm{day}$ and a switch to sunitinib directly in patients with an exon 11 nondeletion mutation.

IM: Imatinib; PFS: Progression-free survival.

When patients in the IM escalation and sunitinib groups were compared in those with an exon 11 deletion mutation, the median PFS in the sunitinib group was significantly longer than in the IM escalation to $600 \mathrm{mg} /$ day period
(16 months; 95\% CI: $1.5-30.5$ vs 3 months; $95 \%$ CI: $2.5-3.5$, respectively; $\mathrm{p}<0.001)$ and a shift to sunitinib therapy after IM escalation period (16 months; $95 \%$ CI: $1.5-30.5$ vs 8 months; 95\% CI: $0.4-15.6$, respectively; $\mathrm{p}=0.024)$ in 
IM escalation group (Figure 2A \& B). However, when patients in the two groups were compared in those with an exon 11 nondeletion mutation, the median PFS in the sunitinib group was not significantly different to that in the IM escalation to $600 \mathrm{mg} /$ day period (9 months; $95 \% \mathrm{CI}$ : 5.6-12.4 vs 5 months; 95\% CI: 3.6-6.4, respectively; $\mathrm{p}=0.011$ ) and a shift to sunitinib therapy after IM escalation period (9 months; $95 \%$ CI: 5.6-12.4 vs 9 months; 95\% CI: 5.6-12.4, respectively; $\mathrm{p}=0.212$ ) in the $\mathrm{IM}$ escalation group (Figure 2 C \& D).

\section{- Toxicity}

All the 38 patients whose IM dosage escalated to $600 \mathrm{mg} /$ day had Grade 1 or 2 adverse events including edema, fatigue, nausea, granulocytopenia and anemia, among which 7 suffered Grade 3 fatigue (7.9\%) and 4 suffered Grade 3 granulocytopenia (10.5\%). In the patients who received sunitinib therapy, common adverse events consisted of hypertension, hand-foot syndrome, stomatitis, hypothyroidism, proteinuria, granulocytopenia, thrombocytopenia and anemia, most of which were Grade 1 or 2 . However, 14 patients had Grade 3 adverse events including hand-foot syndrome $(8 \%, 6 / 75)$, proteinuria $(1.3 \%, 1 / 75)$ and granulocytopenia $(6.7 \%$, 5/75).

\section{Discussion}

IM dose escalation and sunitinib are both recommended as treatment options after the failure of first-line standard-dose IM therapy in patients with advanced GIST [11,12]. Selection of the optimal therapy in these patients has been suggested to depend on the mutation type present. Although it has been reported that patients with GIST who have exon 9 mutations and wild-type phenotypes can obtain more benefit from sunitinib therapy than those with exon 11 mutations, two Phase III studies of IM dose escalation failed to identify the $c$-kit/PDGFRA genotype subgroups who may benefit from this approach $[6,13]$. As a result, it is difficult to formulate a comprehensive treatment plan and properly sequence the two therapies.

A retrospective study from Taiwan which included 91 patients with metastatic GIST who had failed on first-line IM treatment found no differences in the PFS or OS between IM dose escalation and sunitinib therapy [14]. However, another retrospective study reported that switching to sunitinib directly achieved a better
PFS than IM dose escalation (10 vs 5 months, respectively) [10], which was a similar finding to our study. We found that switching to sunitinib therapy directly achieves a longer PFS compared with IM dose escalation after the failure of firstline IM treatment. Although some patients in the IM dose escalation group of our study may have received sunitinib prior to the occurrence of disease progression, the PFS with IM dose escalation only (3 months) was similar to the PFS reported in two Phase III studies (81 days, 5 months) [5,6] and our previous study in Chinese patients with GIST (17 weeks) [15]. In addressing the question as to whether it is better to give IM dose escalation therapy followed by sunitinib or switch to sunitinib directly after the failure of standard-dose IM therapy, we found that while switching to sunitinib directly achieved a significantly longer PFS (14 vs 9 months, respectively; $\mathrm{p}=0.006$ ), there was no significant difference in OS between the two approaches (21 vs 20 months, respectively; $\mathrm{p}=$ $0.230)$.

We also evaluated the efficacy of sunitinib in different $c$-kit gene exon 11 mutation genotypes in this study and found that patients with an exon 11 deletion mutation had a trend toward a better PFS compared with patients with an exon 11 nondeletion mutation. However, this phenomenon was not apparent with IM dose escalation therapy which may be due to the fact that the clinical benefit of IM dose escalation is related to the increased blood concentration achieved [16] rather than to genotype differences in efficacy. Another important finding of the present study was that the median PFS of patients with an exon 11 deletion mutation was significantly longer in the sunitinib group than in the IM dose escalation group (16 vs 8 months, respectively; $\mathrm{p}=0.024$ ), although there was no difference in PFS between the two groups in patients with an exon 11 nondeletion mutation. This finding suggests that patients with GIST who have an exon 11 deletion mutation should switch to sunitinib directly after the failure of standard-dose IM therapy.

\section{Conclusion}

In conclusion, switching to sunitinib directly after the failure of first-line IM therapy is associated with an improved PFS in comparison with IM dose escalation followed by subsequent sunitinib therapy. Patients with GIST who have an exon 11 deletion mutation are more likely to 
benefit from switching to sunitinib therapy after the failure of first-line IM therapy than from escalating the dose of IM.

Financial \& competing interests disclosure

The authors have no relevant affiliations or financial involvement with any organization or entity with a financial interest in or financial conflict with the subject matter or materials discussed in the manuscript. This includes employment, consultancies, honoraria, stock ownership or options, expert testimony, grants or patents received or pending, or royalties.
Editorial assistance with the manuscript was provided by Content Ed Net, Shanghai Co. Ltd.

Ethical conduct of research

Approval for the study was given by the Beijing Cancer Hospital Ethics Committee.

\section{Open access}

This work is licensed under the AttributionNonCommercial-NoDerivatives 4.0 Unported License. To view a copy of this license, visit http://creativecommons.org/ licenses/by-nc-nd/4.0/

\section{SUMMARY POINTS}

- There is no consensus on whether imatinib (IM) dose escalation followed by subsequent sunitinib therapy or switching to sunitinib directly after the failure of first-line IM treatment in advanced or metastatic gastrointestinal stromal tumor (GIST) patients and whether there are differences in the efficacy of second-line sunitinib therapy in patients with different exon 11 mutation genotypes.

- A total of 75 patients with advanced or metastatic GIST were analyzed retrospectively to compare the efficacy of second-line sunitinib therapy in patients with exon 11 deletion and nondeletion mutations, and to compare the efficacy of IM dose escalation followed by sunitinib with switching to sunitinib directly after failure of first-line IM treatment.

- Among the 75 patients, 38 received IM dose escalation followed by sunitinib (IM escalation group), while 37 were switched to sunitinib directly after the failure of first-line IM treatment (sunitinib group). Disease control rates in the sunitinib and IM escalation groups were 78.4 and $44.7 \%$, respectively $(p=0.002)$. Over a median follow-up period of 56 months, the median progression-free survival (PFS) in the sunitinib group was significantly longer than in the IM escalation group (14 vs 4 months; $p<0.001$ ). In patients with exon 11 deletions, the median PFS was significantly longer in the sunitinib group than in the IM escalation group (16 vs 3 months; $p<0.001$ ).

- In patients with advanced or metastatic GIST who progress on standard-dose IM therapy, switching to sunitinib directly was associated with an improved PFS in comparison with IM dose escalation followed by subsequent sunitinib therapy.

- Patients with GIST who have an exon 11 deletion mutation are more likely to benefit from switching to sunitinib therapy than from escalation of the IM dose after failure of standard-dose IM therapy.

\section{References}

1 Zhao X, Yue C. Gastrointestinal stromal tumor. J. Gastrointest. Oncol. 3(3), 189-208 (2012).

2 Miettinen M, Lasota J. Gastrointestinal stromal tumors (GISTs): definition, occurrence, pathology, differential diagnosis and molecular genetics. Pol. J. Pathol. 54(1), 3-24 (2003).

3 Demetri GD, von Mehren M, Blanke CD et $a l$. Efficacy and safety of imatinib mesylate in advanced gastrointestinal stromal tumors. N. Engl. J. Med. 347(7), 472-480 (2002).

4 Lee JH, Kim Y, Choi JW et al. Correlation of imatinib resistance with the mutational status of KIT and PDGFRA genes in gastrointestinal stromal tumors: a meta- analysis. J. Gastrointestin. Liver Dis. 22(4), 413-418 (2013).

5 Blay JY, Le Cesne A, Ray-Coquard I et al. Prospective multicentric randomized Phase III study of imatinib in patients with advanced gastrointestinal stromal tumors comparing interruption versus continuation of treatment beyond 1 year: the French Sarcoma Group. J. Clin. Oncol. 25(9), 1107-1113 (2007).

6 Blanke CD, Rankin C, Demetri GD et al. Phase III randomized, intergroup trial assessing imatinib mesylate at two dose levels in patients with unresectable or metastatic gastrointestinal stromal tumors expressing the kit receptor tyrosine kinase: S0033. J. Clin. Oncol. 26(4), 626-632 (2008).
7 Demetri GD, van Oosterom AT, Garrett CR et al. Efficacy and safety of sunitinib in patients with advanced gastrointestinal stromal tumour after failure of imatinib: a randomised controlled trial. Lancet 368(9544), 1329-1338 (2006).

8 Heinrich MC, Maki RG, Corless CL et al. Primary and secondary kinase genotypes correlate with the biological and clinical activity of sunitinib in imatinib-resistant gastrointestinal stromal tumor. J. Clin. Oncol. 26(33), 5352-5359 (2008).

9 Cho S, Kitadai Y, Yoshida S et al. Deletion of the KIT gene is associated with liver metastases and poor prognosis in patients with gastroinetstinal stromal tumors in the stomach. Int. J. Oncol. 28(6), 1361-1367 (2006). 
(nenzi B, Nannini M, Fumagalli E et al. Imatinib dose escalation versus sunitinib as a second line treatment in KIT exon 11 mutated GIST: a retrospective analysis. Oncotarget 7(43), 69412-69419 (2016).

11 Chinese Gastrointestinal Stromal Tumor Expert Committee, CSCO. Consensus on diagnosis and treatment for gastrointestinal stromal tumor in China (2013 version). Chin. Clin. Oncol. 18(11), 1025-1032 (2013).

12 National Comprehensive Cancer Network. NCCN Clinical Practice Guidelines in
Oncology: GIST, v1 (2015).

www.nccn.org/professionals

13 Zalcberg JR, Verweij J, Casali PG et al.

Outcome of patients with advanced gastro-intestinal stromal tumours crossing over to a daily imatinib dose of $800 \mathrm{mg}$ after progression on $400 \mathrm{mg}$. Eur. J. Cancer 41(12), 1751-1757 (2005).

14 Hsu CC, Wu CE, Chen JS et al. Imatinib escalation or sunitinib treatment after first-line imatinib in metastatic gastrointestinal stromal tumor patients. Anticancer Res. 34(9), 5029-5036 (2014).

15 Li J, Gong JF, Li J et al. Efficacy of imatinib dose escalation in Chinese gastrointestinal stromal tumor patients. World J. Gastroenterol. 18(7), 698-703 (2012).

16 Widmer N, Decosterd L, Csajka C et al. Imatinib plasma levels: correlation with clinical benefit in GIST patients. Br. J. Cancer 102(7), 1198-1199 (2010). 